
About IJMA [last updated July, $\left.1^{\text {st }}, 2021\right]$

$\checkmark$ International Journal of Medical Arts is the Official Journal of the Damietta Faculty of Medicine, AlAzhar University, Egypt

$\checkmark$ It is an International, Open Access, Double-blind, Peer-reviewed Journal

$\checkmark$ Published four times a year

$\checkmark$ The First Issue was published in July 2019

$\checkmark$ Published under the following license: Creative Commons Attribution-ShareAlike 4.0 International Public License (CC BY-SA 4.0). It had updated from the Creative Commons license [CC BY] in volume 2, Issue 4, October 2020 About IJMA

$\checkmark$ The Egyptian Knowledge Bank hosts the web site of IJMA

$\checkmark$ The Egyptian Knowledge Bank supports IJMA

$\checkmark$ IJMA follows the regulations of the International Committee of Medical Journal Editors

$\checkmark$ IJMA is indexed in the "Directory of Open Access Journals" [15 January 2021].

$\checkmark$ IJMA is indexed in JGate [29-6-2021]

$\checkmark$ IJMA is a member of the International Society of Managing and Technical Editors

$\checkmark$ Listed in "Index Copernicus", "Publons", "Academic resource index [ResearchBib]", "Electronics journal library", "Eurasian Scientific Journal Index", and "Citefactor"

$\checkmark$ IJMA introduced to the search engine [BASE] through DOAJ
Click image to reach the page

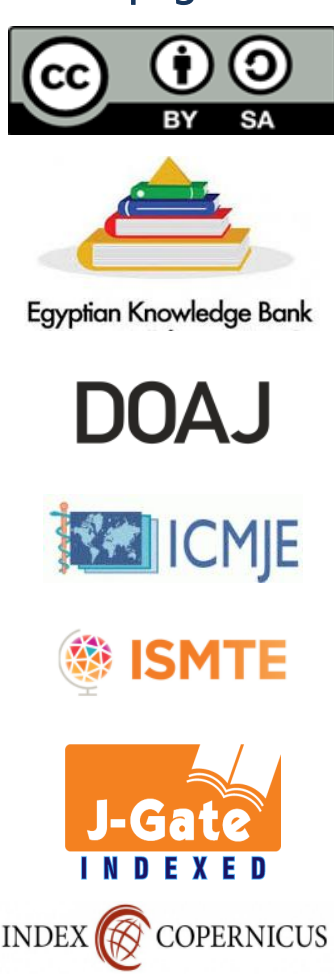

publons

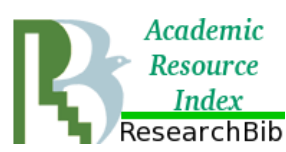

\section{EZ3 \\ .}

ESJII

CiteFactor

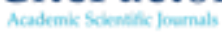

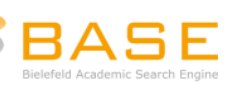




Available online at Journal Website
https://ijma.journals.ekb.eg/
Main subject [Cardiology]

Original Article

\title{
Assessment of Left Ventricular Systolic Function by Speckle Tracking in Diabetic Patients Receiving Direct Acting Antiviral Drugs for Chronic HCV
}

\author{
Wael Anwar Hassib [1]; Mohamed Gamil Abd Elraouf [1]; Reda Biomy [1]; Mohammed A. Hamouda [2]; \\ Mohamed Mahrous Ali Mohamed [2] \\ ${ }^{1}$ Department of Cardiology, Faculty of Medicine, Kafrelsheikh University, Egypt. \\ 2 Department of Cardiology, Faculty of Medicine, Benha University, Egypt.
}

Corresponding author: Wael Anwar Hassib

Email:wael372002@yahoo.com

Submission date: April 13, 2021; Acceptance date: July 23, 2021

DOI: $10.21608 / / J M A .2021 .80445 .1326$

Background: Diabetes mellitus [DB] is a well-known risk factor for cardiovascular disease. The co-existence of DM and microvascular abnormalities could lead to hypertrophy of left ventricle [LV] with diastolic and/or systolic dysfunction. However, early stages of the disease are asymptomatic due to its chronic nature and the effective control of diabetes.

Aim of the Work: This clinical cohort aimed to assess left ventricular systolic function in DM patients, receiving direct acting antiviral [DAA] drugs for chronic hepatitis- $C$ virus by speckle tracking.

Patients and Methods: This clinical cohort included 90 patients who were divided into three equal groups. Patients were recruited from Hepatology Outpatient Clinic of Kafr El-Shaikh University hospital, who were sent to the cardiovascular department for evaluation. Patients were evaluated just before, one month and three months after starting therapy.

Result: No one in control group had diastolic dysfunction compared to $10.0 \%$ and $16.7 \%$ in DAAs and Non-DAAs groups, respectively. Conventional echocardiography returned statistically non-significant difference except significant increase of LVESD, IVST and LVPWT in study groups than control groups. However, the difference between DAAs and Non-DAAs groups was statistically non-significant. The basic strain parameters returned significant lower values at different windows in control than study groups. The strains were reduced in the follow up visits [first and third months] in DAAs groups, but not significantly reduced in non-DAAs group. Acute myocardial infarction was reported among one patient in non-DAAs group compared to none in DAAs group.

Conclusion: The speckle tracking is a useful tool for early detection of subclinical left ventricular dysfunction in HCV diabetic patients and could be used for following up of response DAA treatment. DAAs therapy is associated with improvement, not of HCV disease, but also of cardiac dysfunction.

Keywords: Diabetes Mellitus; Left Ventricle; Hepatitis C Virus; Echocardiography; Direct anti-viral drugs

This is an open-access article registered under the Creative Commons, ShareAlike 4.0 International license [CC BY-SA 4.0] [https://creativecommons.org/licenses/by-sa/4.0/legalcode.

Citation: Hassib WA, Abd Elraouf MG, Biomy R, Hamouda MA, Mohamed MMA. Assessment of Left Ventricular Systolic Function by Speckle Tracking in Diabetic Patients Receiving Direct Acting Antiviral Drugs for Chronic HCV. IJMA 2020; 3[3]: July-September: $1694-1699$ [DOI: 10.21608/IJMA.2021.80445.1326].

* Main subject and any subcategories have been classified according to the research topic. 


\section{INTRODUCTION}

Diabetes mellitus [DM] is a well-known risk factor for cardiovascular diseases. The coexistence of DM and microvascular changes could lead to hypertrophy of the left ventricle [LV] and diastolic or systolic dysfunction. However, in the early duration of the disease, the patient is usually asymptomatic due to chronic nature of DM, especially if associated with strict control. Echocardiography could detect non-specific abnormalities of the left ventricle function. However, conventional echocardiography usually does not recognize subclinical changes ${ }^{[1]}$.

Chronic hepatitis $\mathrm{C}$ virus [HCV] infection is a common infection worldwide, as it affects $>3 \%$ of total populations. It could be associated with or lead to a significant complication such as liver cirrhosis or hepatocellular carcinoma ${ }^{[2]}$.

In addition, $\mathrm{HCV}$ infection is endemic in Egypt, with the highest prevalence rate in all over the world. In 2015, a National Egyptian survey was completed, and the prevalence of HCV was $7.0 \%$ in populations aged $15-59$ years ${ }^{[3]}$.

The treatment of HCV included peg interferon-alfa and ribavirin [RBV]. However, its efficacy is limited and associated with higher rate of side effects. New drugs included direct acting antivirals [DAAs] drugs with higher efficacy and safety profile especially on the myocardium ${ }^{[4]}$.

DAAs target specific non-structural proteins of the hepatitis-C virus and so, lead to interruption of viral replication and thus eradicate infection. DDAs composed of various categories according to their therapeutic target and mechanism of action. The first is nonstructural proteins 3/4A [NS3/4A] protease inhibitors [PIs] [e.g., Glecaprevir, Paritaprevir, Voxilaprevir, Galexos, Grazoprevir, and Sunvepra]. The second is Nucleoside and Nucleotide NS5B polymerase inhibitors [NPIs] [e.g., Sofosbuvir]. The third is NS5B non-nucleoside polymerase inhibitors [NNPIs] [e.g., Dasabuvir], and the fourth is NS5A inhibitors [e.g., Daclatasvir, Elbasvir, Ledipasvir, Ombitasvir, Velpatasvir Ombitasvir, and Pibrentasvir] ${ }^{[5]}$.

The potential cardiac side effects of DAAs in a retrospective study of 34 patients were cardiac dysfunction among 14 patients [41.2\%]; six patients had reduced [<30.0\%] left ventricle ejection fraction, and eight had LVEF $30-50 \%$; 11 patients need hospitalization. These side effects lead to clinical withdrawal of BMS-986094 [6].

For accurate assessment of global and regional myocardial deformation, the speckle tracking echocardio- graphy [STE] had been emerged as an accurate technique on the quantitative basis $[7,8]$.

STE assessment of myocardial deformation allows the identification of subclinical left ventricular dysfunction in diseases appeared normal by conventional echocardiography [9].

The rationale of the current work based on the above truth, where speckle tracking could be used to elucidate the subclinical myocardial dysfunction. This allows early intervention and prevention of development of clinical cardiac dysfunction. This protects our patients, improve their quality of life, and reduce the total healthcare cost.

\section{THE AIM OF THE WORK}

This study aimed to evaluate the role of speckle tracking in assessment of left ventricular systolic function in diabetic patients, receiving direct acting antiviral [DAA] drugs for chronic hepatitis-C virus infection.

\section{PATIENTS AND METHODS}

The current study was designed as an observational, prospective, clinical trial that included 90 patients who were divided into three equal groups [each group composed of 30 patients].

The first group included 30 healthy persons as a control group. The second group included 30 diabetic patients with chronic HCV who received direct acting antiviral drugs. The third group was 30 diabetic patients with chronic HCV with no antiviral treatment [they refused administration of any antiviral drugs and just accepted tonics and liver support].

All patients were recruited from Hepatology Outpatient Clinic of Kafr El-Shaikh University hospital who refereed to cardiovascular department of the same hospital for evaluation. The inclusion criteria were adult diabetic patients with chronic hepatitis $\mathrm{C}$ virus [HCV] starting direct acting antivirals [DAA] for the first time. On the other side, the exclusion criteria were cardiomyopathy, myocardial infarction within the last three months, and relapse of earlier antiviral therapy.

\section{Ethical considerations:}

The study protocol was approved by the local research and ethics committee of Kafr El Shaikh Faculty of Medicine, and an informed consent was signed by every patient. Patient's rights to withdraw at any time without any harm were guaranteed; and the study had been completed 
according to research ethics code declared at Helsinki.

Patients were evaluated just before inclusion, at one and three months after starting therapy by the following: full medical history stressing on cardiac symptoms, full clinical examination, and Investigations [Electrocardiography, Conventional two-dimensional and M-Mode Echocardiography-Doppler and Speckle tracking]. The standard 12 leads ECG was done for all patients and controls to detect cardiac rhythm, chamber enlargement and to exclude ischemic changes. The conventional, tissue Doppler and two-dimensional speckle tracking echocardiography were performed using S5-1 probe of Philips Epic 7c machine.

All echocardiographic examinations were performed according to the recommendations of American Society of Echocardiography [ASE] and European Association of Cardiovascular Imaging [EACVI].

By conventional echocardiography, left ventricular diameters and wall thicknesses were measured in the left parasternal long axis view at the level of the mitral valve tips, ensuring a measurement perpendicular to the long axis of the ventricle. On the remote side, the speckle tracking is an offline technique that applied to previously acquired 2D images. Longitudinal strain was measured using software on 2D gray-scale images of LV from standard apical four-chamber, two-chamber and threechamber views. The peak systolic [PS] global longitudinal strain [GLS] was calculated as the average of the LS of the $17 \mathrm{LV}$ segments obtained from $4-\mathrm{CH}, 2-\mathrm{CH}$, and $3-\mathrm{CH}$ views. Global longitudinal Strain [GLS] was used to detect subclinical left ventricular systolic dysfunction.

\section{Statistical analysis:}

Data collected from history, clinical examination, laboratory investigations and outcome measures were coded, entered, and analyzed using Microsoft Excel software. Data were then imported into Statistical Package for the Social Sciences [SPSS version 20.0] [IBM ${ }^{\circledR} S P S S{ }^{\circledR}$ Inc., Chicago, Illinois, USA] software for analysis. According to the type of data, qualitative data were represented as frequencies and percentages. However, quantitative continues data were represented by mean \pm standard deviation [SD]. The following tests were used to test differences or associations for significance; Chi square test [X2] for qualitative data and independent groups by one way analysis of variance with post-Hoc least significant differences [LSD] for comparison between two groups and paired by paired [t] test and repeated ANOVA for comparison over time.

\section{RESULTS}

In the current work, studied groups were comparable regarding patient's age and gender. The mean age was $54.6 \pm 7.6,53.63 \pm 6.8$ and $53.13 \pm 7.5$ years in control, DAAs and non-DAAs groups, respectively. Males represented $53.3 \%, 63.3 \%$ and $53.3 \%$ of control, DAAs and non-DAAs groups, respectively [Table 1].

The diastolic dysfunction did not differ significantly between studied groups. The diastolic dysfunction was absent in control group, $10 \%$ in non-DAAs group and $16.7 \%$ in DAAs group. However, it was indeterminate in $0.0 \%, 6.7 \%$ and $10.0 \%$ in control, non-DAAs and DAAs groups, respectively. Otherwise, it was normal among $100.0 \%, 83.3 \%$ and $73.3 \%$ in control, non-DAAs and DAAs groups, respectively.

Regarding conventional echocardiography, studied groups showed statistically non-significant difference except significant increase of LVESD, IVST and LVPWT in study groups when compared to control group. However, the difference between DAAs and Non-DAAs groups was statistically non-significant. On the other side, each of septal e' and lateral e' were significantly higher among control than study groups. However, average E/e' and LAVI was significantly lower in control than study groups [Table 2].

Regarding strain parameters in the first visit, there was significant difference between study and control groups in the values at $A 4 C, A 3 C, A 2 C$ and global longitudinal strain [GLS]. The control group had the lower values, then DAAs and Non-DAAs groups, respectively [Table 3]

At the first visit [one month after treatment], speckle tracking strains were significantly lower among DAAs than non-DAAs group. The comparable situation was reported at three months [Table 4]. In DAAs group, there was no significant change in strain values from the first to the third visit. However, in non-DAAs group, the A4C, A3C and GLS significantly changed at the third visit when compared to the corresponding values at the first visit [Table 5].

Regarding Ml, there were no cases of Ml in normal and DAAs groups. However, there was one patient [3.3\%] of non-DAAs group. There was no significant difference between studied groups $[p=0.36$ ] 


\begin{tabular}{|l|l|c|c|c|c|}
\hline \multicolumn{7}{|c|}{ Table [1]: Comparison between groups, about patient age and gender } \\
\hline Age & Control [n=30] & DAAs [n=30] & Non- DAAs [n=30] & $P$ \\
\hline \multirow{2}{*}{ Sex } & Female & $54.6 \pm 7.6$ & $53.63 \pm 6.8$ & $53.13 \pm 7.5$ & 0.734 \\
\cline { 2 - 5 } & Male & $14[46.7 \%]$ & $11[36.7 \%]$ & $14[46.7 \%]$ & 0.66 \\
\hline
\end{tabular}

Table [2]: Comparison between groups, about conventional echocardiography, and diastolic function parameters

\begin{tabular}{|c|c|c|c|c|c|}
\hline & & Control [n=30] & Diabetic group & & $P$ \\
\hline & & & DAAs $[n=30]$ & Non-DAAs [n=30] & \\
\hline Conventional & LVEDD [cm] & $4.66 \pm 0.39$ & $4.92 \pm 0.48$ & $4.84 \pm 0.36$ & 0.053 \\
\hline Echocardiography & LVESD [cm] & $2.86 \pm 0.29 \#$ & $3.11 \pm 0.35$ & $3.05 \pm 0.32$ & $0.014^{*}$ \\
\hline parameters & EF [\%] & $68.36 \pm 3.5$ & $66.06 \pm 4.6$ & $67.96 \pm 4.6$ & 0.094 \\
\hline & $\mathrm{FS}[\%]$ & $37.53 \pm 3.05$ & $35.96 \pm 3.8$ & $37.4 \pm 4.39$ & 0.212 \\
\hline & IVST [cm] & $0.96 \pm 0.12^{\#}$ & $1.02 \pm 0.09$ & $1.02 \pm 0.08$ & $0.036^{*}$ \\
\hline & LVPWT [cm] & $0.97 \pm 0.11^{\#}$ & $1.02 \pm 0.08$ & $1.03 \pm 0.07$ & $0.040^{*}$ \\
\hline Diastolic & E wave & $82.9 \pm 12.4$ & $84.9 \pm 16.3$ & $77.91 \pm 21.6$ & 0.273 \\
\hline Function & A wave & $67.67 \pm 11.3$ & $67.59 \pm 13.01$ & $74.46 \pm 13.2$ & 0.057 \\
\hline parameters & E/A ratio & $1.26 \pm 0.3$ & $1.32 \pm 0.4$ & $1.11 \pm 0.35$ & 0.120 \\
\hline & Septal e' & $10.26 \pm 1.9^{\&}$ & $8.22 \pm 1.77$ & $8.49 \pm 1.7$ & $<0.001^{*}$ \\
\hline & Lateral e' & $12.99 \pm 3.12^{8}$ & $11.05 \pm 2.16$ & $11.08 \pm 1.67$ & $0.002^{*}$ \\
\hline & Aver E/e' & $7.12 \pm 0.98^{\#}$ & $9.11 \pm 1.75$ & $8.08 \pm 2.5$ & $<0.001^{*}$ \\
\hline & TR velocity & $2.33 \pm 0.24$ & $2.2 \pm 0.3$ & $2.28 \pm 0.36$ & 0.290 \\
\hline & LAVI & $27.64 \pm 3.7^{\#}$ & $29.6 \pm 2.3$ & $31.42 \pm 1.9$ & $<0.001^{*}$ \\
\hline
\end{tabular}

LVEDD: left ventricle end-diastolic volume; LVESD: left ventricle end-systolic volume, EF: ejection fraction, FS: fraction shortening, IVST: interventricular septal thickness, LVPWT, left ventricle posterior wall thickness, TR: Tricuspid, LAVl; Left atrial maximum volume index; *: significant difference between groups; \#: significant decrease in control when compared to DAAs or non-DAAs groups.

Table [3]: Comparison between strain parameters among groups at the first visit

\begin{tabular}{|l|c|c|c|c|}
\hline \multirow{2}{*}{} & Control group & \multicolumn{2}{|c|}{ Diabetic patients } & \multirow{2}{*}{$P$} \\
\cline { 3 - 4 } & & DAAs [n=30] & Non-DAAs [n=30] & \\
\hline A4C & $-20.93 \pm 1.3$ & $-20.06 \pm 1.2$ & $-18.56 \pm 1.2$ & $<0.001^{*}$ \\
\hline A3C & $-20.52 \pm 1.2$ & $-19.86 \pm 1.23$ & $-18.42 \pm 1.4$ & $<0.001^{*}$ \\
\hline A2C & $-21.28 \pm 1.5$ & $-19.6 \pm 1.01$ & $-18.74 \pm 1.2$ & $<0.001^{*}$ \\
\hline GLS & $-20.91 \pm 1.2$ & $-19.8 \pm 0.99$ & $-18.55 \pm 1.1$ & $<0.001^{*}$ \\
\hline
\end{tabular}

Table [4]: Shows comparison between DAAs and non-DAAs groups

\begin{tabular}{|l|l|c|c|c|}
\hline & & DAAs [n=30] & Non DAAs [n=30] & $P$ \\
\hline At one month & A4C & $-20.06 \pm 1.13$ & $-18.27 \pm 1.13$ & $<0.001^{*}$ \\
\cline { 2 - 5 } & A3C & $-19.9 \pm 1.12$ & $-17.96 \pm 1.25$ & $<0.001^{*}$ \\
\cline { 2 - 5 } & A2C & $-19.6 \pm 0.9$ & $-18.67 \pm 1.25$ & $0.002^{*}$ \\
\cline { 2 - 5 } & GLS & $-19.84 \pm 0.91$ & $-18.26 \pm 0.99$ & $<0.001^{*}$ \\
\hline \multirow{4}{*}{ At three months } & A4C & $-20.02 \pm 1.26$ & $-17.78 \pm 1.5$ & $<0.001^{*}$ \\
\cline { 2 - 5 } & A3C & $-19.8 \pm 1.18$ & $-17.68 \pm 1.6$ & $<0.001^{*}$ \\
\cline { 2 - 5 } & A2C & $-19.5 \pm 0.82$ & $-18.39 \pm 1.67$ & $0.002^{*}$ \\
\cline { 2 - 5 } & GLS & $-19.77 \pm 0.94$ & $-17.91 \pm 1.42$ & $<0.001^{*}$ \\
\hline
\end{tabular}

Table [5] Comparison between strain results before and after antiviral treatment in DAAs and non-DAAs groups.

\begin{tabular}{|l|l|l|l|l|l|l|l|}
\hline Group & & \multicolumn{2}{|c|}{ First visit } & \multicolumn{2}{c|}{ Third visit } & test & $p$ \\
\hline \multirow{4}{*}{ DAAs } & A4C & -20.06 & 1.26 & -20.02 & 1.26 & -0.51 & 0.608 \\
\cline { 2 - 8 } & A3C & -19.86 & 1.23 & -19.80 & 1.18 & -0.81 & 0.423 \\
\cline { 2 - 8 } & A2C & -19.60 & 1.01 & -19.50 & .82 & -0.86 & 0.392 \\
\cline { 2 - 8 } & GLS & -19.84 & .99 & -19.77 & .94 & -1.07 & 0.294 \\
\hline \multirow{4}{*}{ Non-DAAs } & A4C & -18.56 & 1.28 & -17.78 & 1.54 & -4.096 & $<0.001^{*}$ \\
\cline { 2 - 8 } & A3C & -18.42 & 1.46 & -17.68 & 1.63 & -3.55 & $0.001^{*}$ \\
\cline { 2 - 8 } & A2C & -18.74 & 1.19 & -18.39 & 1.67 & -1.47 & 0.15 \\
\cline { 2 - 8 } & GLS & -18.55 & 1.15 & -17.91 & 1.42 & -3.63 & $0.001^{* *}$ \\
\hline
\end{tabular}




\section{DISCUSSION}

The current work aimed to assess left ventricular systolic function in diabetic patients receiving direct acting antiviral [DAA] treatment for chronic HCV by conventional and two-dimensional speckle tracking echocardiography, for early detection of subclinical left ventricular systolic dysfunction in this group of patients before overt clinical diabetic cardiomyopathy ensues.

In our study, diabetic patients had lower significant values of longitudinal strain parameters [AP4C LS, AP2C LS, AP3C LS, GLS] than control group. These results agree with Perticone et al. ${ }^{[10]}$ who reported that, $\mathrm{HCV}$, normotensive patients when compared to healthy control have a significant echocardiographic changes and increased cardiac mass. These changes could add to explanation of pathogenic mechanisms of cardiovascular morbidity associated with chronic hepatitis $\mathrm{C}$ infection.

There is available evidence that, left ventricular hypertrophy is a powerful predictor of comorbid cardiovascular events, in general populations and in different chronic clinical diseases [11]. Insulin resistance plays a participating role in the development of left ventricular hypertrophy ${ }^{[12]}$. Earlier studies also revealed that, HCV infection is associated with the development of insulin resistance, through direct interaction between viral products and different signaling pathways of insulin $[13,14]$. The eradication of HCV infection induced a significant reduction of insulin resistance and could prevent the development of type-2 diabetes [15, 16]. Early detection of associated cardiovascular abnormalities in HCV infection associated with type-2 diabetes mellitus is crucial for patients' outcome. The concomitant presence of both conditions adds to the risk of morbidity and mortality. In addition, the eradication of HCV infection reduced both the risks of insulin resistance and cardiovascular morbidity. Thus, early initiation of anti-viral therapy is of utmost importance. Additionally, lorga et al. [17] reported that, although chronic HCV infection significantly increased the risk to the development of $\mathrm{T} 2 \mathrm{D}$, the prediction to develop $\mathrm{T} 2 \mathrm{D}$ is still under investigation

The echocardiographic results of the current study showed both HCV-diabetic groups at inclusion in the study. However, control group had significantly lower left ventricle end systolic diameter, lower thickness of interventricular septum and posterior wall thickness of the left ventricle. Otherwise, no difference was elicited about end diastolic volume, ejection fraction and fraction shortening. Additionally, six out of eight diastolic function parameters returned non-significant difference between diabetics and healthy controls. On the contrary, the strain parameters by speckle tracking revealed significant changes in diabetic than healthy controls. Additionally, after 3 months treatment regimens, strain parameters revealed significant improvement in DAAs than non-DAAs groups. These results reflected the ability of speckle tracking to detect LV abnormalities and to follow up the response of treatment. Finally, only one patient developed acute coronary syndrome in non-DAAs group, reflecting high safety profile of DAAs.

The idea after speckle tracking based on its ability to measure the displacement of speckles on the twodimensional images, measures the deformation of the myocardium rather than its volumetric change measured by traditional LVEF determination methods [18]. Potter and Marwick [19] assessed the left ventricular function by echocardiography, included global longitudinal strain, and appreciated its role in the early detection of diastolic or systolic dysfunction. In addition, the early detection of LVD before the onset of cardiac symptoms enables the proper use of drugs at a point, which may slow or prevent disease progression. Adinolfi et al. [20] showed that, the risk of cardiovascular incidents [e.g., acute coronary syndrome or $\mathrm{TIA}]$ were significantly reduced by DAAs treatment were decreased and annual incidence of HCV was highly reduced. They added DAAs are characterized by a favorable safety profile. Biomy et al. [21] confirmed the safety of DAAs in an Egyptian study, where DAAs therapy did not cause any change in QTc interval, in patients with or without liver cirrhosis. Another later study from Egypt confirmed such safety profile [22]. Roguljic et al. [23] returned the reduction of cardiovascular risk to an improvement of endothelial function and glucose metabolism. Ibrahim et al. [22] concluded that, HCV infection treatment with DAAs used in Egyptians has a good cardiac safety profile. It had no effect on QTc interval, left or right ventricular functions except for a reduction in right ventricle GLS in those with no liver cirrhosis.

On the extreme side, Farrag et al. [24] reported cardiotoxic effects of DAAs on the myocardium, in both patients with normal and impaired basic ejection fraction. However, they included 100 patients with impaired and only 20 patients with normal basic ejection fraction. They did not find significant difference when stratifying their patients according to their gender, different chronic disease states [e.g., hypertension, diabetes, ischemic heart disease, and established cardiac medications]. This heterogeneity in their study design could explain the contradictory nature of their results in comparison to the current one. 
Conclusion: Diabetes mellitus could be considered as a latent, independent risk factor for cardiac dysfunction, which may progress to heart failure. HCV infection is a systemic disease with extra-hepatic manifestations, which could be detected before the diagnosis of HCV itself. Diabetic groups had significant lower global strain than control group. The speckle tracking is a useful tool for early detection of subclinical left ventricular dysfunction in HCV diabetic patients and could be used for following up of response DAA treatment. DAAs therapy is associated with improvement, not of HCV disease, but also of cardiac dysfunction.

Financial and Non-financial Relationships and Activities of Interest: None to be declared

\section{REFERENCES}

1. Sohrabi C, Saberwal B, Lim WY, Tousoulis D, Ahsan S, Papageorgiou N. Heart Failure in Diabetes Mellitus: An Updated Review. Curr Pharm Des. 2020; 26[46]:5933-5952. [DOI: 10.2174/ 1381612826666201118091659].

2. Mohd Hanafiah K, Groeger J, Flaxman AD, Wiersma ST. Global epidemiology of hepatitis $C$ virus infection: new estimates of agespecific antibody to HCV seroprevalence. Hepatology. 2013 Apr; 57 [4]: 1333-42. [DOI: 10.1002/ hep.26141].

3. Gomaa A, Allam N, Elsharkawy A, El Kassas M, Waked I. Hepatitis C infection in Egypt: prevalence, impact, and management strategies. Hepat Med. 2017 May 15; 9:17-25. [DOI: 10.2147/HMER.S113681].

4. Liang TJ, Ghany MG. Current, and future therapies for hepatitis $C$ virus infection. N Engl J Med. 2013 May 16; 368 [20]:1907-17. [DOI: 10.1056/NEJMra1213651].

5. Poordad F, Dieterich D. Treating hepatitis C: current standard of care and emerging direct-acting antiviral agents. J Viral Hepat. $2012 \mathrm{Jul}$; 19[7]:449-64. [DOI: 10.1111/j.1365-2893.2012.01617. x].

6. Ahmad T, Yin P, Saffitz J, Pockros PJ, Lalezari J, Shiffman M, et al. Cardiac dysfunction associated with a nucleotide polymerase inhibitor for treatment of hepatitis C. Hepatology. 2015 Aug; 62[2]: 409-16. [DOI: 10.1002/hep.27488].

7. Mondillo S, Galderisi M, Mele D, Cameli M, Lomoriello VS, Zacà V, et al., and Echocardiography Study Group of The Italian Society of Cardiology [Rome, Italy]. Speckle-tracking echocardiography: a new technique for assessing myocardial function. J Ultrasound Med. 2011; 30[1]:71-83. [DOI: 10.7863/jum.2011.30.1.71].

8. Fontana A, Zambon A, Cesana F, Giannattasio C, Trocino G. Tissue Doppler, triplane echocardiography, and speckle tracking echocardiography: different ways of measuring longitudinal myocardial velocity and deformation parameters. A comparative clinical study. Echocardiography. 2012 Apr; 29 [4]: 428-37. [DOI: 10.1111/j.15408175.2011.01618. x].

9. Imbalzano E, Zito C, Carerj S, Oreto G, Mandraffino G, CusmàPiccione M, Di Bella G, Saitta C, Saitta A. Left ventricular function in hypertension: new insight by speckle tracking echocardiography. Echocardiography. 2011 Jul; 28 [6]: 649-57. [DOI: 10.1111/j.15408175.2011.01410. x].

10. Perticone M, Miceli S, Maio R, Caroleo B, Sciacqua A, Tassone EJ, et al. Chronic HCV infection increases cardiac left ventricular mass index in normotensive patients. J Hepatol. 2014; 61[4]:755-60. [DOI: 10.1016/j.jhep.2014.05. 032].

11. Stewart MH, Lavie CJ, Shah S, Englert J, Gilliland Y, Qamruddin S, et al. Prognostic Implications of Left Ventricular Hypertrophy. Prog Cardiovasc Dis. 2018; 61[5-6]: 446-455. [DOI: 10.1016/j.pcad. 2018.11.002].

12. Sesti G, Sciacqua A, Scozzafava A, Vatrano M, Angotti E, Ruberto C, Santillo E, Parlato G, Perticone F. Effects of growth hormone and insulin-like growth factor-1 on cardiac hypertrophy of hypertensive patients. J Hypertens. 2007 Feb; 25[2]:471-7. [DOI: 10.1097/ HJH.0b013e3280112b63].

13. Gill M, UI Qamar M, Ikram F, Naz S, Sadaf H, Hafeez Z. Insulin Resistance and Chronic Hepatitis C: Relationship with Serum Iron and Hepcidin. Cureus. 2020 Dec 28; 12[12]: e12349. [DOl: 10.7759/cureus.12349].

14. Perticone M, Maio R, Tassone EJ, Tripepi G, Di Cello S, Miceli S, et al. Insulin-resistance HCV infection-related affects vascular stiffness in normotensives. Atherosclerosis. 2015; 238[1]: 108-12. [DOI: 10.1016/j.atherosclerosis.2014. 11.025].

15. Gastaldi G, Gomes D, Schneiter P, Montet X, Tappy L, Clément S, Negro $F$. Treatment with direct-acting antivirals improves peripheral insulin sensitivity in non-diabetic, lean chronic hepatitis $\mathrm{C}$ patients. PLOS One 2019 Jun 6; 14[6]: e0217751. [DOI: 10.1371/ journal.pone.0217751].

16. Hum J, Jou JH. The link between hepatitis $C$ virus and diabetes mellitus: Improvement in insulin resistance after eradication of hepatitis C virus. Clin Liver Dis [Hoboken]. 2018 Apr 6;11[3]:73-76. [DOI: 10.1002/cld.694].

17. lorga RA, Bacalbasa N, Bratu OG, Ionita Radu F, Diaconu CC. The impact of infection with hepatitis $C$ virus on cardiovascular risk. Am J Cardiovasc Dis. 2020 Aug 15; 10[3]:201-206. [PMID: 32923102].

18. Shah AM, Solomon SD. Myocardial deformation imaging: current status and future directions. Circulation. 2012; 125[2]: e244-8. [DOI: 10.1161/CIRCULATIONAHA.111.086348]

19. Potter E, Marwick TH. Assessment of Left Ventricular Function by Echocardiography: The Case for Routinely Adding Global Longitudinal Strain to Ejection Fraction. JACC Cardiovasc Imaging. 2018; 11[2 Pt 1]:260-274. [DOI: 10.1016/j.jcmg.2017.11.017].

20. Adinolfi LE, Petta S, Fracanzani AL, Nevola R, Coppola C, Narciso $\mathrm{V}$, et al. Reduced incidence of type 2 diabetes in patients with chronic hepatitis $\mathrm{C}$ virus infection cleared by direct-acting antiviral therapy: A prospective study. Diabetes Obes Metab. 2020; 22[12]:2408-2416. [DOI: 10.1111/dom.14168].

21. Biomy R, Abdelshafy M, Abdelmonem A, Abu-Elenin H, Ghaly G. Effect of Chronic Hepatitis C Virus Treatment by Combination Therapy on Cardiovascular System. Clin Med Insights Cardiol. 2017; 11:1179546817713204. [DOI: 10.1177/ 1179546817713204].

22. Ibrahim MG, Sharafeldin AA, Mousa NI, Mousa TK, El Missiri AM. Effect of direct-acting antivirals on corrected QT interval and cardiac functions in patients with chronic hepatitis $\mathrm{C}$ virus infection. Egypt Heart J. 2020 Feb 7; 72[1]: 7. [DOI: 10.1186/s43044-020-0042-y].

23. Roguljic H, Nincevic V, Bojanic K, Kuna L, Smolic R, Vcev A, et al. Impact of DAA Treatment on Cardiovascular Disease Risk in Chronic HCV Infection: An Update. Front Pharmacol. 2021 May 11; 12:678546. [DOI: 10.3389/fphar.2021.678546]

24. Farrag HM, Monir MS, Abdel-Dayem WS, Ali HA, Ibrahim AM. Global longitudinal strain as a predictor of short-term effect of oral antiviral regimens on myocardium in Egyptian patients with chronic viral hepatitis C. Egypt Heart J. 2021 Jan 9; 73[1]:6. [DOI: 10.1186/s43044-020-00129-2]. 

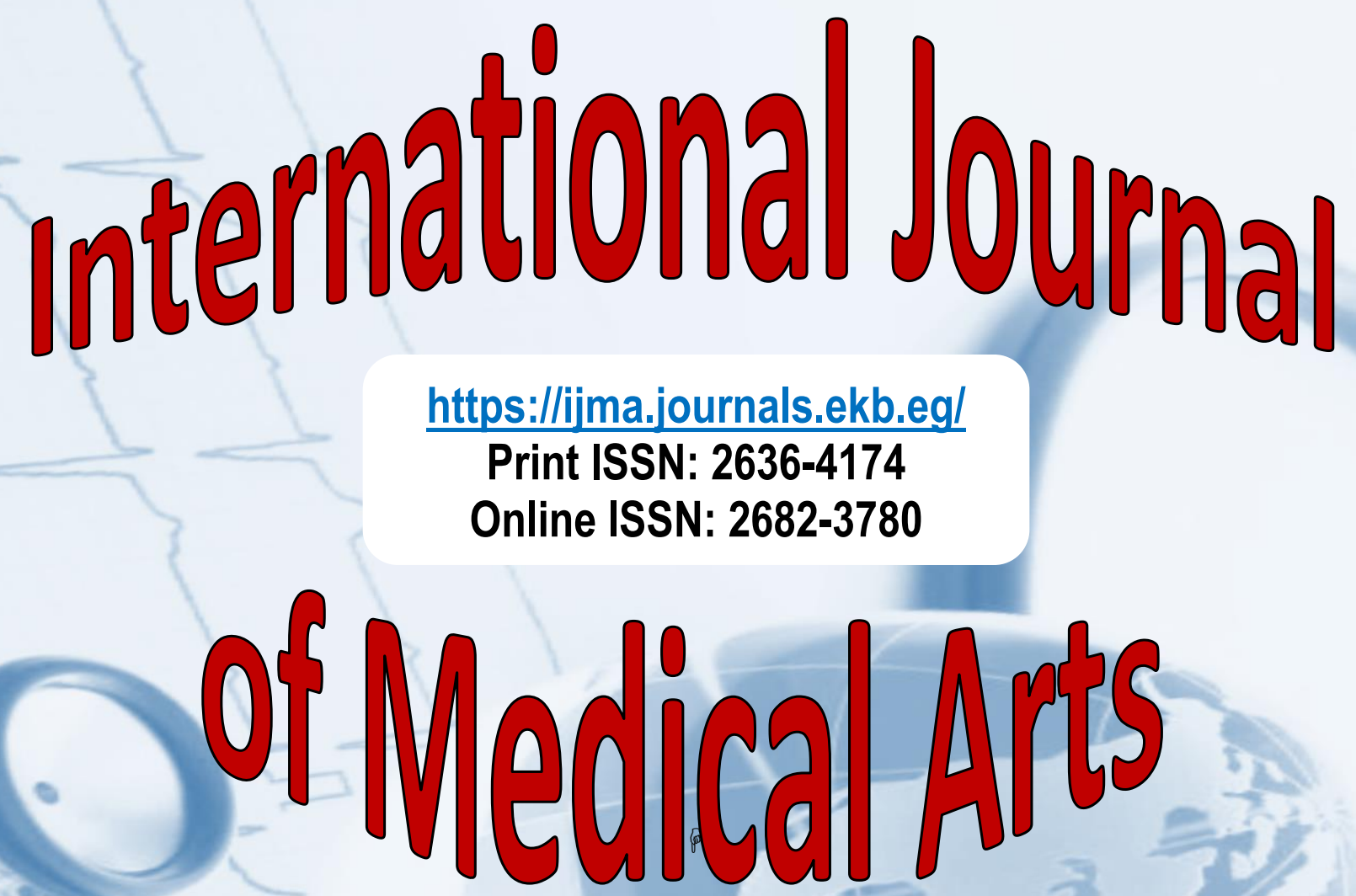